\title{
Comparative assessment of dissipated energy and other fatigue criteria ${ }^{1}$
}

\author{
Alexander M. Korsunsky*a \\ ${ }^{a}$ Department of Engineering Science \\ and Rolls-Royce University Technology Centre (UTC) in Solid Mechanics \\ University of Oxford, Parks Road, Oxford OX1 3PJ, UK \\ *Corresponding author, e-mail alexander.korsunsky@eng.ox.ac.uk \\ Tel. +44 (1865) 273043 Fax +44 (1865) 273010 \\ Daniele Dini $^{\text {a,b }}$ \\ ${ }^{b}$ Current address: Department of Mechanical Engineering, \\ Imperial College, Exhibition Road, London SW7 2AZ, UK \\ Fionn P.E. Dunne ${ }^{a}$ \\ ${ }^{a}$ Department of Engineering Science \\ and Rolls-Royce University Technology Centre (UTC) in Solid Mechanics \\ University of Oxford, Parks Road, Oxford OX1 3PJ, UK \\ Michael J. Walsh \\ ${ }^{c}$ Rolls-Royce plc, P.O. Box 31, Derby DE24 8BJ, UK
}

\begin{abstract}
Amongst a large number of fatigue criteria proposed for the prediction of crack initiation in thermomechanical fatigue, various approaches have been found to be particularly useful for certain categories of material over specific domains of temperature and cyclic strain. However, no particular approach appears to give invariably better predictions than others, so that the choice of the lifing model must be based on validation for the relevant circumstances.

In this paper the focus is placed on the Energy Dissipation Criterion (EDC). We present physical arguments in favour of this approach's versatility, and illustrate its performance by the application of this approach both in the macroscopic and micromechanical context.

Firstly, by way of illustration we consider cyclic deformation of a Ramberg-Osgood material, with a view to establish the equivalence between the EDC and some selected classical criteria. For this simple but analytically tractable case several interesting results can be established, including the equivalence between EDC and both stress range and strain range lifing criteria.

Secondly, we consider fatigue loading of polycrystalline FCC material deforming by the combination of anisotropic linear elasticity and crystal slip. Energy dissipation density in this case is location-dependent even for a polycrystal subjected to macroscopically uniform stress and strain. Crack in itiation then is predicted to occur at the 'weakest link' location corresponding to the most intense dissipation.

The above two versions of energy dissipation criteria are each compared against experimental data. The comparative performance of Walker strain, Smith-Watson-Topper (SWT) and EDC lifing methods is assessed. It is concluded that EDC provides improved reliability, particularly for cases of complex loading paths and mechanisms interactions.
\end{abstract}

Keywords: fatigue, energy dissipation, crack initiation, structural integrity, non-linear deformation

\footnotetext{
${ }^{1}$ Paper presented at the International Conference on Fatigue and Damage of Structural Materials, Hyann is, MA, USA, 17-22 September 2006.
} 


\section{Introduction}

Reliable prediction of fatigue durability of structures remains a major challenge in mechanical engineering design and exploitation (Socie and Marquis [1], Suresh [2]). Applications range widely, from automotive, nuclear, and aeronautical industries to micro- and nano-technology and MEMS/NEMS design. Reliable prediction of fatigue durability requires a combination of several contributing factors: the knowledge of constitutive deformation laws validated across a wide range of temperatures and loading conditions, availability of relevant experimental data, and correct identification of the relationship between deformation history and damage (or, equivalently, residual life). For thermo-mechanical fatigue (TMF) conditions, the constitutive law should allow the prediction of both isothermal and anisothermal deformation and damage across a large range of temperatures and multiaxial stress states, and implementable in the form of suitably efficient numerical tools.

\section{Criteria for fatigue life prediction}

Popular criteria for the prediction of thermo-mechanical fatigue crack initiation include Damage Summation (DS), Frequency Separation (FS), Ductility Exhaustion (DE), Strain-Range Partitioning (SRP), Total-Strain Version of SRP (TS-SRP), Strain Energy Partitioning (SEP), and Energy Dissipation Criteria (EDC) (e.g. Suresh [2]).

Without considering in detail the basis or formulation of the established life prediction criteria and their application to multi-axial thermo-mechanical fatigue, we wish to compare their effectiveness in the prediction of fatigue failure with the approach using dissipated energy as the principal damage parameter.

The general formulation of a fatigue criterion (Constantinescu et al, [3]) can be expressed mathematically as a relation between the number of cycles to failure of the structure $N_{f}$ and some function of the cyclic strain or stress values,

$$
\Phi\left(\varepsilon, \varepsilon_{p l}, \sigma, \ldots\right) N_{f}^{b}=c
$$

where $N_{f}$ denotes the number of cycles to failure, $b, c$ are material dependent constants, and $\boldsymbol{\varepsilon}, \boldsymbol{\varepsilon}_{p l}$,

$\boldsymbol{\sigma}$ are values of the deformation parameter taken at certain points in a (stabilised) cycle. Classical fatigue criteria (e.g. Suresh, [2]) can all be expressed in the above form. For the Manson-Coffin [4, 5], Smith-Watson-Topper [6], Walker [7] and Ostergren criteria [8], the form of function $\boldsymbol{\Phi}$ is given in Error! Reference source not found.. Here $\Delta \boldsymbol{\varepsilon}_{p l}, \boldsymbol{\Delta} \boldsymbol{\varepsilon}, \boldsymbol{\sigma}_{\max }$ denote, respectively, the plastic 
strain range, the total strain range and the maximum tensile stress over a uniaxial cycle; $E$ is the Young modulus and $\mathbf{v}$ the frequency of the test cycle; $b, k, c, m$ are material parameters characterising the fatigue behaviour.

\section{[ Table 1 here ]}

A potential difficulty arises in the choice of the form of function $\Phi$ for multiaxial loading cases. Here the strain range has to be replaced with a well-chosen amplitude of the strain cycle computed using a suitable "norm" (e.g. the von Mises equivalent stress $J_{2}$ ). Similarly, the mean tensile stress over a cycle may have to be replaced by the mean hydrostatic stress when its influence on the lifetime is being considered. While these generalisations may appear suitable for the passage from uniaxial to multiaxial isothermal problems, it is more difficult to justify for multi-axial anisothermal, since over a large range of temperatures the parameters $b, k, c, m$ may vary strongly (Charkaluk and Constantinescu [9]).

Furthermore, formulating the failure criterion in terms of several point-wise values (often taken at the maximum and minimum extreme points in the cycle) clearly presents a limitation. For example, it is possible to imagine an infinite family of fatigue loop shapes which share the same extreme points, but nevertheless have distinctly different appearance, shape and area. A more general formulation would allow $\Phi$ to be a functional, i.e. depend on the deformation history including the combination of stress and strain values at all points in the stabilised loop.

\section{Energy dissipation criterion}

Following Charkaluk et al. [10] and Charkaluk and Constantinescu [9], and earlier work of Skelton and co-workers [11-13], the functional $\boldsymbol{\Phi}$ may be written as:

$$
\Phi(x)=\int_{\text {cycle }} \sigma(x, t) \dot{\varepsilon}(x, t) d t
$$

where the integral is taken over the stabilized cycle, and $\boldsymbol{x}$ denotes the point at which the field values are considered. In order to find the critical location within the structure, the criterion is written as

$$
\left[\max _{x} \boldsymbol{\Phi}(\boldsymbol{x})\right] \boldsymbol{N}_{f}^{b}=c
$$

A particular choice of the form of the functional can be made on the basis that most classical criteria are in fact in some way related to the dissipated energy per cycle, since they combine products of strains and stresses. Skelton $[14,15]$ suggested that the dissipated energy per saturated cycle could 
be considered as a material constant expressing the necessary energy to propagate a crack by the distance equivalent to the size of the process zone, and proposed a way in which the lifetime of the specimen was related to a crack propagation law driven by dissipated energy. Although it is clear that only a fraction of the dissipated energy contributes to damage, it is possible to postulate that this fraction itself also mostly depends on the energy dissipation, thus preserving the possibility of formulating a viable criterion on the basis of th is parameter alone.

In objects of complex shape subjected to anisothermal fatigue the energy dissipation per cycle is a scalar parameter that has to be computed numerically. In the absence of experimental data for all necessary combinations of strain ranges and temperatures this parameter must be estimated using suitably general numerical models adjusted to match the available data.

\section{Energy dissipation in a Ramberg-Osgood material}

In order to elucidate the relationship between the energy-based criterion and other conventional fatigue criteria (e.g. strain-based for LCF, stress-based for HCF, etc.) consider a material for which the monotonic cyclic stress-strain curves obey the Ramberg-Osgood law in the form:

$$
\varepsilon=\varepsilon_{e l}+\varepsilon_{p l}=\left(\frac{\sigma}{E}\right)+\left(\frac{\sigma}{K}\right)^{1 / n},
$$

where $\varepsilon$ is the total strain, $E$ denotes Young's modulus and $K$ the hardening modulus, and $n$ is the hardening exponent observed for power law hardening at large strains, when $\sigma=K \varepsilon^{n}$. For each stabilised fatigue cycle between stresses $-\sigma_{\mathrm{m}}$ and $\sigma_{\mathrm{m}}$ and strains between $-\varepsilon_{\mathrm{m}}$ and $\varepsilon_{\mathrm{m}}$ the maximum strain range is related to the stress range by:

$$
2 \varepsilon_{m}=\left(\frac{2 \sigma_{m}}{E}\right)+\left(\frac{2 \sigma_{m}}{K}\right)^{1 / n} .
$$

Including the offset stress of $-\sigma_{\mathrm{m}}$ and offset strain of $-\varepsilon_{\mathrm{m}}$ allows the loop shape to be described by

$$
\varepsilon=\left(\frac{2\left(\sigma+\sigma_{m}\right)}{E}\right)+\left(\frac{2\left(\sigma+\sigma_{m}\right)}{K}\right)^{1 / n}-\varepsilon_{m}=\frac{\sigma}{E}+\left(\frac{\sigma+\sigma_{m}}{K}\right)^{1 / n}-2^{(1+n) / n}\left(\frac{\sigma_{m}}{K}\right)^{1 / n} \text {. }
$$

The loops shapes for different stress and strain ranges are illustrated in Figure 1(a), and the loop shape difference between two distinct strain ranges is illustrated in Figure 1(b).

To calculate the dissipated energy per cycle as a function of stress and strain range, consider the line connecting the extreme points of the cycle, the equation of which is simply given by

$$
\varepsilon=\varepsilon_{m}\left(\frac{\sigma}{\sigma_{m}}\right)
$$

Calculation of energy dissipation over two halves of the loop area leads to the formula: 


$$
\Delta W=2^{(1+n) / n} \sigma_{m}\left(\frac{1-n}{1+n}\right)\left(\frac{\sigma_{m}}{K}\right)^{1 / n}=\left(2 \sigma_{m}\right)^{(1+n) / n}\left(\frac{1-n}{1+n}\right)\left(\frac{1}{K}\right)^{1 / n} .
$$

The above expression establishes the equivalence of energy dissipation criterion and Basquin's law of stress-based lifing: energy dissipation density $\Delta W$ exhibits power law dependence on the maximum cyclic stress $\sigma_{\mathrm{m}}$, with the exponent explicitly expressed in terms of the Ramberg-Osgood power law exponent, $n$. The same conclusion is drawn for Coffin-Manson law based on plastic strain range, since for Ramberg-Osgood material the relationship between plastic strain range and stress range (and hence total dissipated energy) is also given by a power law in equation (4).

[Figure 1 here]

\section{Numerical experiment}

In this section we consider some numerical experiments aimed at drawing comparisons regarding the predictive capabilities of different fatigue lifing criteria. One of the difficulties arising in this situation has already been mentioned, since the choice of 'correct' fatigue lifing criterion is not in fact known. For the model case of analytically tractable Ramberg-Osgood material it is nevertheless possible to postulate the validity of one of the criteria (total strain range, stress range, SmithWatson-Topper, or energy dissipation), and explore the implications for the relationships between the number of cycles to initiation and the parameters used in other criteria. This numerical experiment is considered in this section.

Figure 1(b) illustrates the family of S-N curves obtained in the assumption of validity of the energy dissipation criterion. Note that here and below the concept of S-N curve is used in the 'generalised' sense, i.e. as a correlation plot between a loading parameter (stress, total strain, dissipated energy, etc.) and the number of cycles to failure. Dissipated energy per cycle is related by a multi-scaling power law to the total strain range and Smith-Watson-Topper (SWT), i.e. undergoes a transition between power law regimes corresponding to low cycle fatigue (LCF) and high cycle fatigue (HCF). Clearly, no fatigue limit is expected for the case of Ramberg-Osgood material, since the transition between elastic and plastic behaviour occurs continuously across the entire strain range. An interesting observation can be made on the basis of Figure 1(b) concerning the fact that the energy-based lifing curve on the diagram shows an appreciably steeper slope than other criteria, offering greater robustness against numerical errors, better resolution, and improved predictive accuracy. 


\section{Microscopic form of the energy dissipation criterion}

In classical fatigue lifing criteria the material is considered to be elastically and plastically isotropic and structure-less. In practice, however, most structural alloys are used in polycrystalline form, i.e. as aggregates of large numbers of small grains of varying orientations. When a polycrystal is subjected to external loading, individual grains deform differently due to the anisotropy of elastic (stiffness) and plastic (crystal slip) properties. Plastically stronger grains carry larger than average stresses, while softer neighbouring grains undergo ready crystal slip and accumulate plastic strain. Upon the removal of external load plastically deformed grains find themselves 'mis-fitting' with respect to their neighbours, giving rise to microscopic residual stress.

The inhomogeneity of deformation at the microscopic level and the attendant residual stresses provide the physical basis for the fatigue criterion due to Dang Van [16]. This criterion postulates that infinite fatigue life (fatigue limit) corresponds to the requirement that elastic shakedown occurs everywhere within the object. Otherwise, energy dissipation continues to take place, and eventually crack initiation must be expected. The shortcoming of the Dang Van criterion lies in the fact that it is expressed in terms of macroscopic parameters, and thus fails to make full use of the physically attractive micro-mechanical hypothes is.

The formulation of micro-mechanically-based energy dissipation criteria for fatigue crack initiation requires the development of an elasto-plastic model of polycrystalline deformation. We list below the features of such model that the authors consider crucial:

- The formulation must be three-dimensional to reflect the three-dimensional nature of crystal deformation by plastic slip

- Grain-level assignment elastic moduli must be anisotropic, to reflect intergranular stiffness mismatch that is likely to affect strain inhomogeneity and load partitioning within the polycrystal

- Microstructure assignment with in the numerical model must be flexible to allow ready modification to match grain size and texture statistics of the polycrystal

- Numerical realisation of the model must be sufficiently economical to allow fast repeated computation to probe the probabilistic and statistical nature of deformation in real polycrystalline materials 
The choice of modelling tools available for the task is not particularly broad, mainly consisting of the mean field self-consistent models (e.g. Korsunsky et al, [17]), and finite element formulations (e.g. Manonukul and Dunne, [18]). The latter FE-based approach to elasto-plastic deformation by crystal slip modelling is used in the present study, with important modifications including threedimensionality and elastic anisotropy of the formulation.

A fixed hexahedral mesh of the representative volume element (RVE) of the polycrystal is used. A microstructure 'implementation' used in conjunction with this mesh is generated using three-dimensional Voronoï tesselation. The grain structure is described by assigning the crystal orientation at each integration point such that it is inherited from the nearest 'seed' point. These seeds collectively represent the origins of grain growth during crystallisation. Controlling the volume density and spatial distribution of the seeds allows microstructural parameters (grain size distribution, grain shape) to be varied. Control of texture, i.e. the statistics of grain orientation distribution function (ODF) is controlled by the choice of orientations prescribed at 'seed points', and can be varied from random to strong by the choice of generating algorithm. It is convenient to refer to a particular set of 'seed' point positions together with prescribed crystal orientations as a 'microstructure implementation'.

The technique described above gives advantage of providing a flexible description of the polycrystalline structure. Crucially, large numbers of microstructure implementations can be readily generated. Once the deformation model has been calibrated, a number of realisations (e.g. variation of texture) can be studied without further experimental input; and probabilistic analyses can be carried out within the crystal plasticity framework.

[Figure 2 here]

Numerical simulations of elasto-plastic polycrystal deformation of idealised nickel-base superalloy material predict strain inhomogeneity and localisation illustrated in Figure 2, where (a) grain orientation representation within the FE model is shown, and (b) contours of accumulated crystal slip are illustrated. Clearly, points where significant slip localisation takes place are also associated with increased dissipation of energy, and would be expected to become initiation sites for fatigue cracks.

The contribution to dissipated en ergy at each load increment can be written as

$$
d W(\mathbf{x})=\sum_{m=1}^{M} \tau_{m} \dot{\gamma}_{m} d t
$$


where $M$ is the total number of active slip systems, $\tau_{m}$ is the current critical resolved shear stress on the $m$-th slip system, $\dot{\gamma}_{m}$ is the shear strain rate, $d t$ is the duration of load increment, and $\mathbf{x}$ refers to the location (integration point). The en ergy dissipated per cycle at point $\mathbf{x}$ is obtained by integrating the above expression, and numerically by summation over the load increments constituting a complete cycle.

\section{Comparison of EDC predictions with experiment}

Low cycle fatigue results analysed were for a nickel-base superalloy used in the manufacture of aeroengine components. For each of the tests the loading conditions, temperature, material properties and the number of cycles to failure were recorded. In the LCF regime the number of cycles to initiation and failure are found to be close, and are used here interchangeably.

Figure 3 shows the comparison between the S-N curves obtained using the Smith-Watson Topper (SWT) criterion and the macroscopic energy dissipation per cycle parameter computed on the basis of experimental stress-strain loop shape data. For both criteria the relationship with the number of cycles to failure is reasonably well approximated by a straight line on the bi-logarithmic plot, i.e. a power law function. One should also observe that a better EDC correlation might be achieved if a function different from the simple power law were to be used. We note, however, that the slope of the energy dissipation criterion (EDC) S-N curve steeper. This implies better sensitivity to small changes of the loading parameter in terms of predicted number of cycles to failure. In our opinion this provides additional justification for making EDC a preferred option for fatigue life prediction from the viewpoint of practical application for design.

Microscopic energy-dissipation criterion was used to obtain a prediction for the S-N curve shown in Figure 4, together with the experimental data indicated by the markers. The dissipated energy per cycle at the most critical point in the simulation was used to obtain this correlation.

\section{Discussion}

Macroscopic and microscopic energy dissipation criteria (EDC) have been analysed in terms of their suitability as the tools for the prediction of fatigue crack initiation and fatigue life under the conditions of initiation domination. Physical arguments for macroscopic en ergy-based approaches to fatigue due to Skelton [11-15], and for microscopic fatigue analysis due to Dang Van [16] have been reviewed. Macroscopic energy dissipation criterion was compared with other well-established 
parameters used for fatigue durability analysis, and found to provide comparable level of predictive ability.

The microscopic energy dissipation criterion was analysed in conjunction with the threedimensional, elastically anisotropic, fixed mesh finite element simulation of polycrystal plasticity. The predictions for fatigue life obtained using this approach were compared with experimental results, and showed good correlation and sensitivity. Energy dissipation per cycle provides a convenient parameter for correlating fatigue life, since it can usually be estimated to good accuracy, both experimentally and within the model.

\section{References}

1. Socie, D. and Marquis, G., Multiaxial fatigue. 2000, Warrendale, PA: Society of Automotive Engineers, 484p.

2. Suresh, S., Fatigue of materials. 1998, Cambrid ge: Cambridge University Press, 679p.

3. Constantinescu, A., Charkaluk, E., Lederer, G., and Verger, L., A computational approach to thermomechanical fatigue. International Journal of Fatigue, 2004. 26(8): p. 805-818.

4. Manson, S.S., Behavior of materials under conditions of thermal stress. 1954 Natl Advisory Comm. on Aeronautics, Report 1170. Cleveland: Lewis Flight Propulsion Laboratory.

5. Coffin, L.F., A study of the effects of cyclic thermal stresses on a ductile metal. Transactions of the American Society of Mechanical Engineers, 1954. 76: p. 931-50.

6. Smith, K.N., Watson, P., and Topper, T.H., Stress-Strain Function for Fatigue of Metals. Journal of Materials, 1970. 5(4): p. 767-\&.

7. Doner, M., Brian, K.R., and Adams, J.H., Evaluation of methods for the treatment of mean stress effects on low-cycle fatigue. Journal of Engineering for Power, 1981: p. 1-9.

8. Ostergren, W.J., Damage Function and Associated Failure Equations for Predicting Hold Time and Frequency Effects in Elevated-Temperature, Low-Cycle Fatigue. Journal of Testing and Evaluation, 1976. 4(5): p. 327-339.

9. Charkaluk, E. and Constantinescu, A., An energetic approach in thermomechanical fatigue for silicon molybdenum cast iron. Materials at High Temperatures, 2000. 17(3): p. 373-380.

10. Charkaluk, E., Bign onnet, A., Constantin escu, A., and Van, K.D., Fatigue design of structures under thermomechanical loadings. Fatigue \& Fracture of Engineering Materials \& Structures, 2002. 25(12): p. 1199-1206.

11. Skelton, R.P. and Loveday, M.S., A re-interpretation of the BCR/VAMAS low cycle fatigue intercomparison programme using an energy criterion. Materials at High Temperatures, 1997. 14(1): p. 53-68.

12. Skelton, R.P., Rees, C.J., and Webster, G.A., Energy damage summation methods for crack initiation and growth during block loading in high-temperature low-cycle fatigue. Fatigue \& Fracture of Engineering Materials \& Structures, 1996. 19(2-3): p. 287-297.

13. Skelton, R.P., Vilhelmsen, T., and Webster, G.A., Energy criteria and cumulative damage during fatigue crack growth. In ternational Journal of Fatigue, 1998. 20(9): p. 641-649.

14. Skelton, R.P., Energy Criterion for High-Temperature Low-Cycle Fatigue Failure. Materials Science and Technology, 1991.7(5):p. 427-439.

15. Skelton, R.P., Cyclic Hardening, Softening, and Crack-Growth During High-Temperature Fatigue. Materials Science and Technology, 1993. 9(11): p. 1001-1008. 
16. Dang-Van, K., Macro-micro approaches in high-cycle multiaxial fatigue. In: McDowell DL, Ellis R, editors. Advances in multiaxial fatigue. Philadelphia (PA): American Society for Testing and Materials; 1993. p. 120-30.

17. Korsunsky, A.M., James, K.E. and Daymond, M.R., Intergranular stresses in polycrystalline fatigue: diffraction measurement and self-consistent modelling. Engineering Fracture Mechanics 71 (2004): p. 805-812.

18. Manonukul, A. and Dunne, F.P.E., High- and low-cycle fatigue crack initiation using polycrystal plasticity. Proceedings of the Royal Society of London Series a- Mathematical Physical and Engineering Sciences, 2004. 460(2047): p. 1881-1903. 


\section{Captions: Table and Figures}

Table 1. This Table presents the values of parameter $\boldsymbol{\Phi}$ used in classical fatigue criteria to build correlations (simple power law or otherwise) against the number of cycles to failure (or to initiation in Low Cycle Fatigue).

Figure 1. (a) Stress-strain plots for a hypoth etical Ramberg-Osgood material subjected to fully reversed tension-compression cyclic deformation $(R=-1)$ with increasing values of maximum stress parameter (denoted $S \_$max on the graph). The cyclic hardening curve is also indicated.

Figure 1. (b) Illustration of correlations between loading parameter and number of cycles to failure for the hypothetical Ramberg-Osgood material. The horizontal axis indicates the number of cycles to failure (log scale), while the vertical axis indicates the values (log scale) of loading parameters used in various criteria. It is assumed, for the purpose of the argument, that Energy Dissipation Criterion (EDC) gives a uniform power law correlation against the number of cycles across the range of conditions considered (filled symbols). The graph illustrates the correlations that then arise for the other three criteria considered (total strain range, stress, and Smith-Watson-Topper), indicated by open symbols. It is interesting to note the appearance of two distinct regimes of low strain and high strain behaviour.

Figure 2. Illustration of (a) smoothed out grain orientation representation within the FE model, and (b) contours of accumulated plastic strain due to crystal slip, and the banded appearance of microscopic shear strain localization.

Figure 3. Comparison between the S-N curves obtained using the Smith-Watson Topper (SWT) criterion (square symbols) and the macroscopic energy dissipation per cycle parameter (round symbols) computed on the basis of experimental stress-strain loop shape data.

Figure 4. Illustration of the correlation between micro scopic form of the energy-dissipation criterion and the number of cycles to failure. 


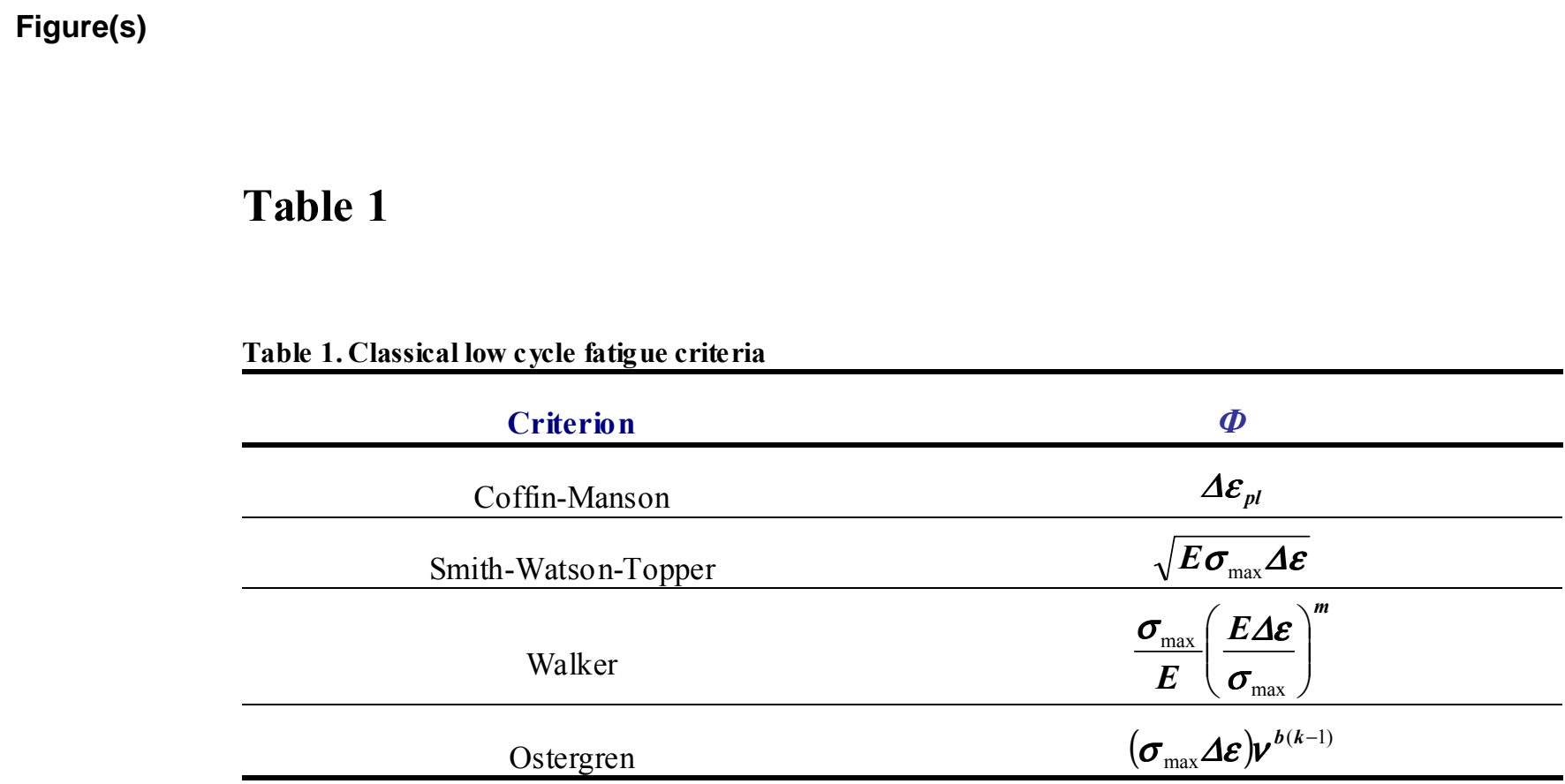

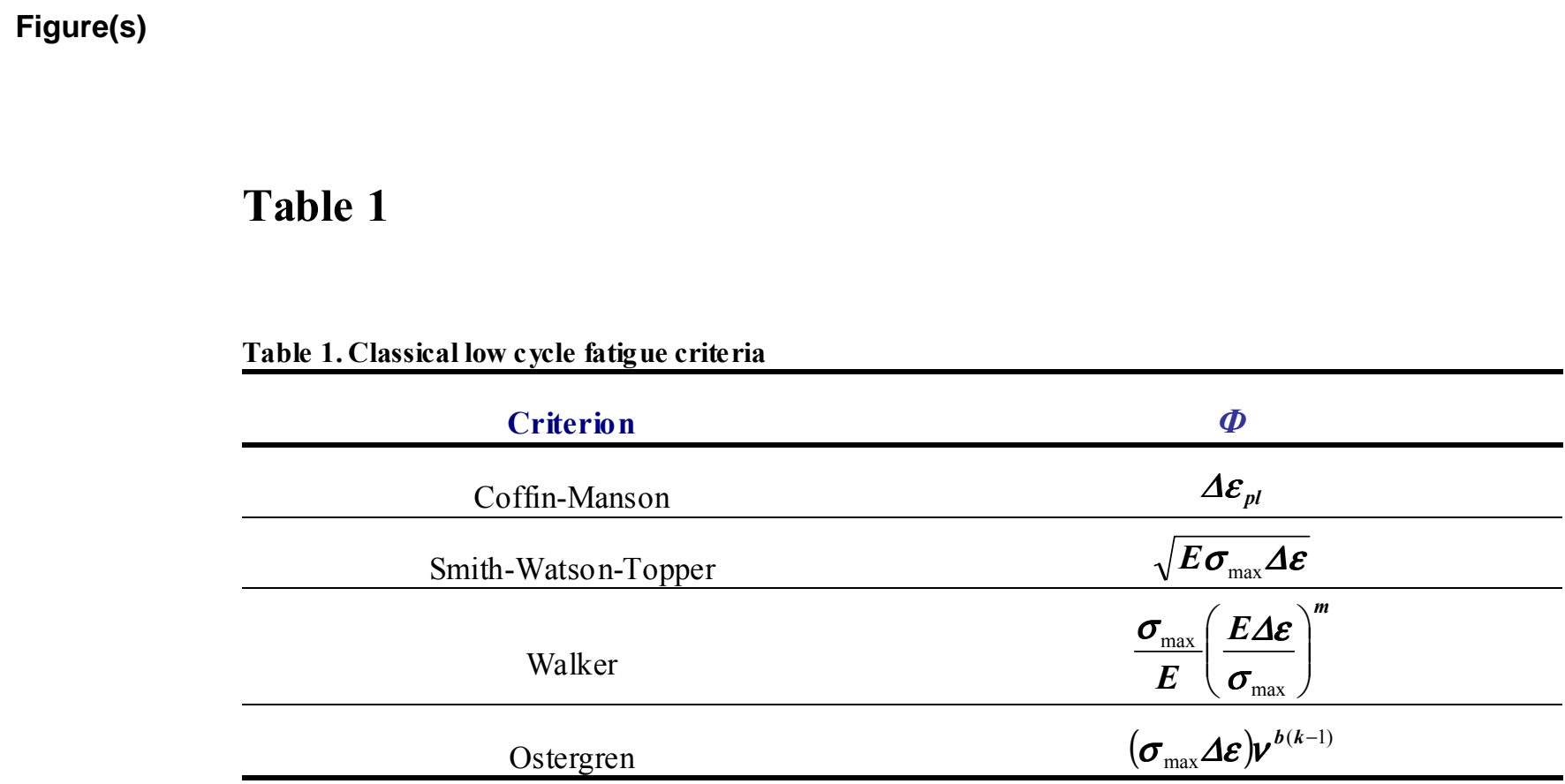

\begin{tabular}{cc}
\hline Criterion & $\Phi$ \\
\hline Coffin-Manson & $\Delta \varepsilon_{p l}$ \\
\hline Smith-Watson-Topper & $\sqrt{E \sigma_{\max } \Delta \varepsilon}$ \\
\hline Walker & $\frac{\sigma_{\max }\left(\frac{E}{E}\left(\frac{\varepsilon}{\sigma_{\max }}\right)^{m}\right.}{\left(\sigma_{\max } \Delta \varepsilon\right) \boldsymbol{v}^{b(k-1)}}$ \\
\hline Ostergren &
\end{tabular}

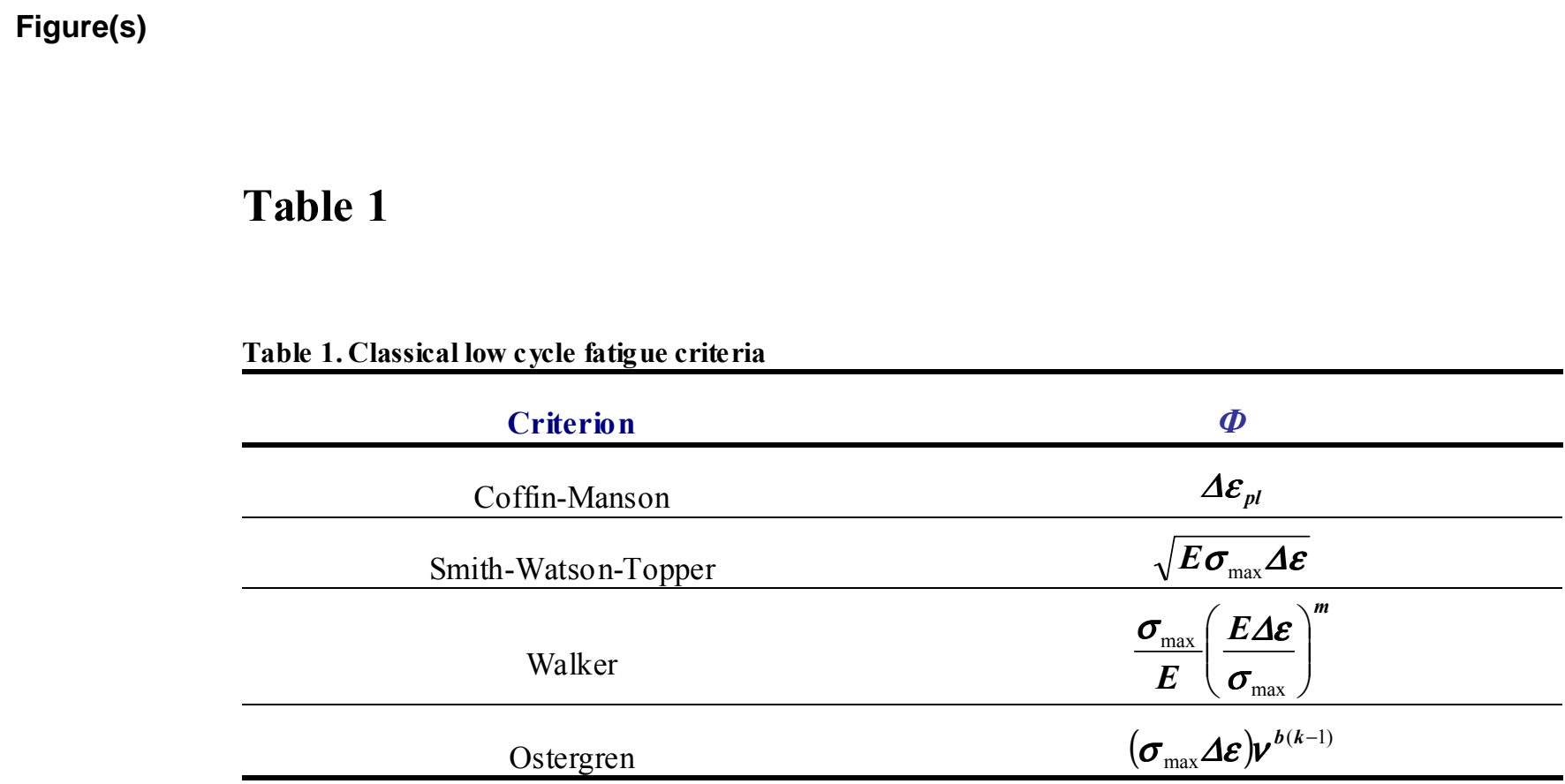

(

政

.

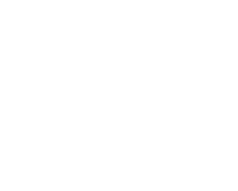




\section{Figures}

(a)

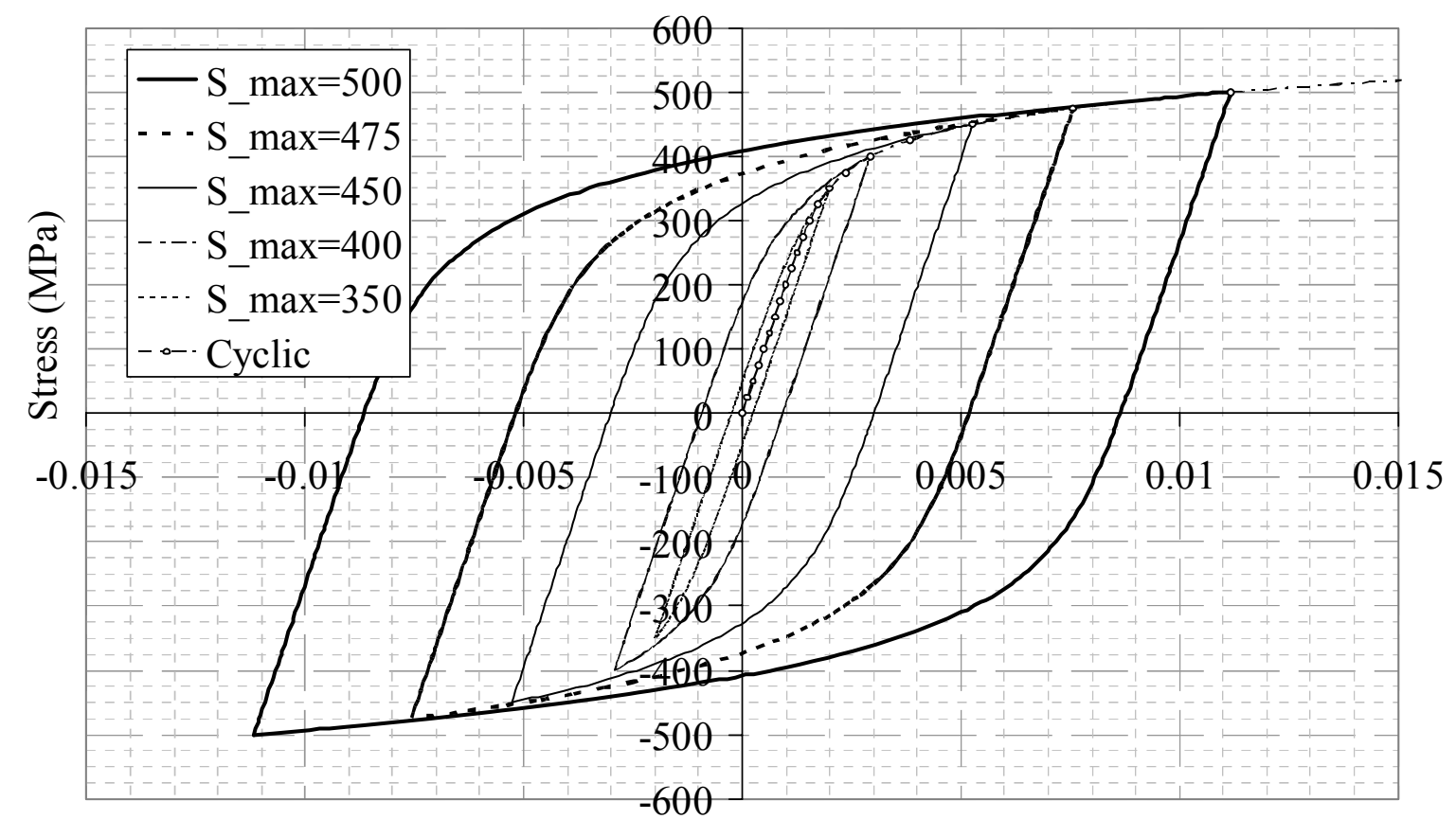

Strain

(b)

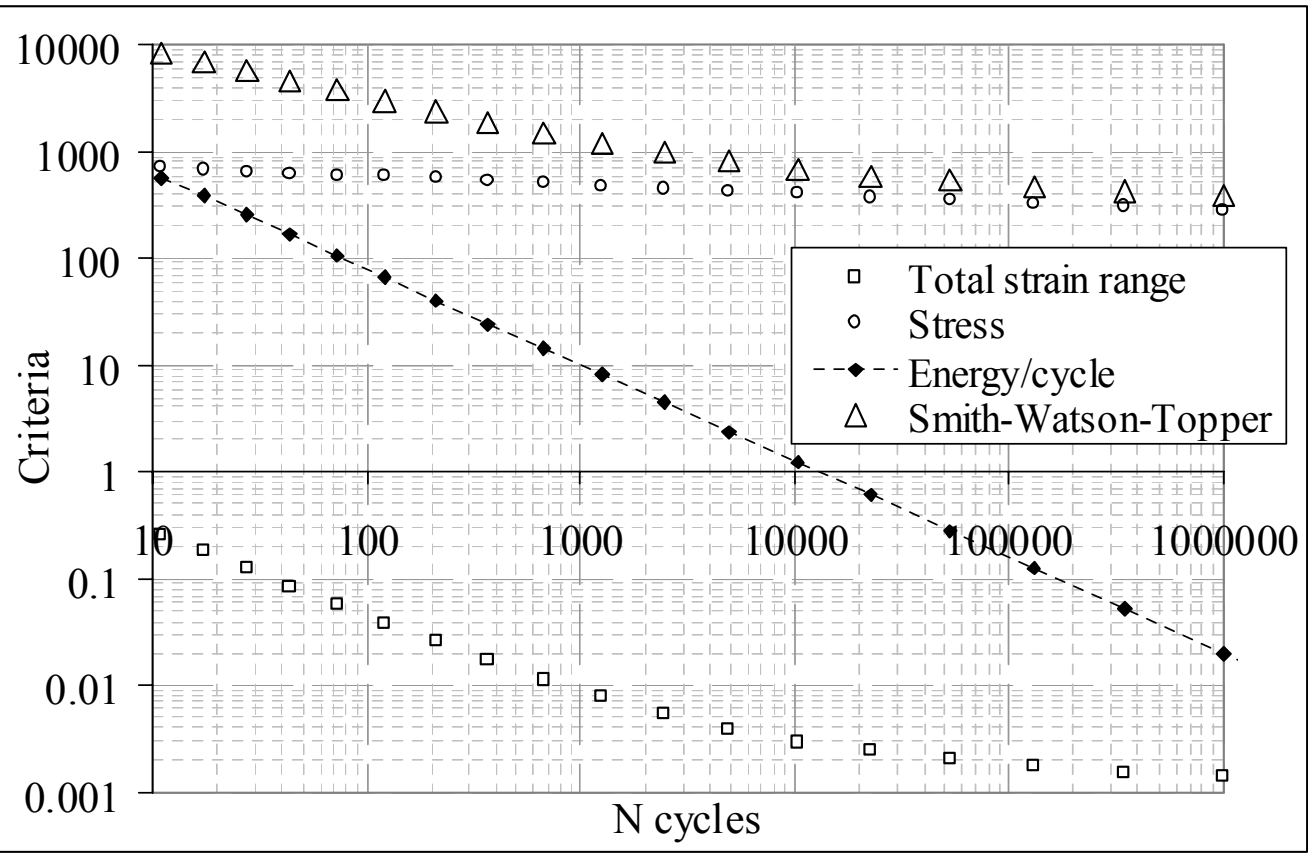

Figure 1. 

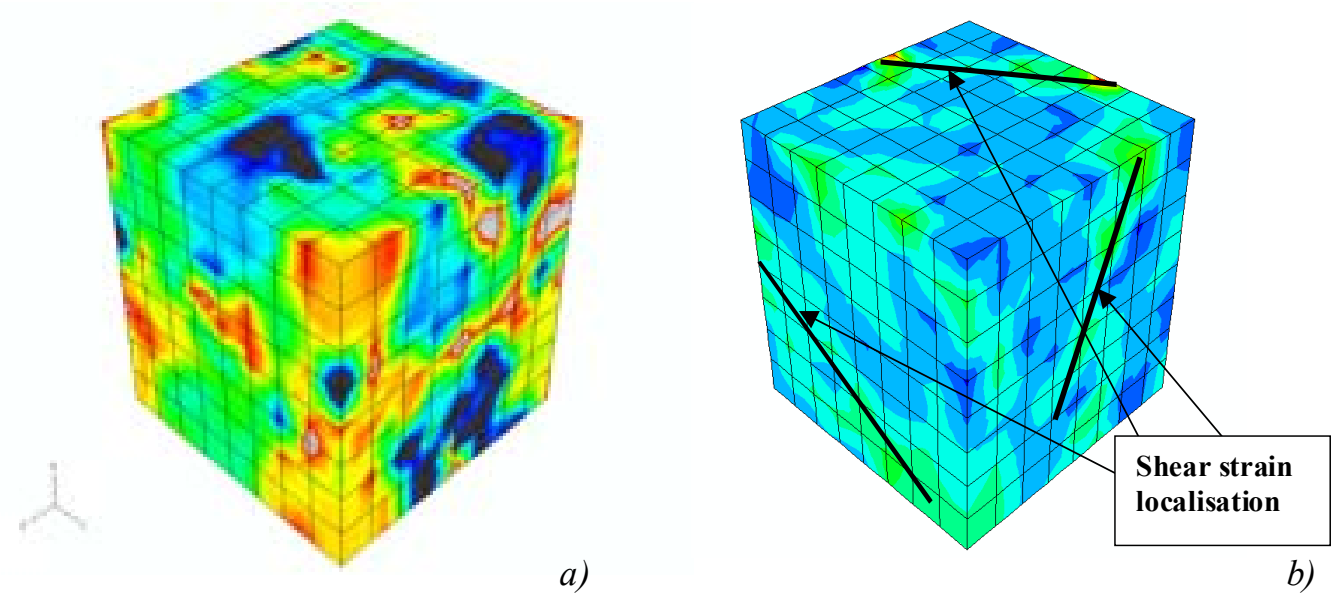

Figure 2. 


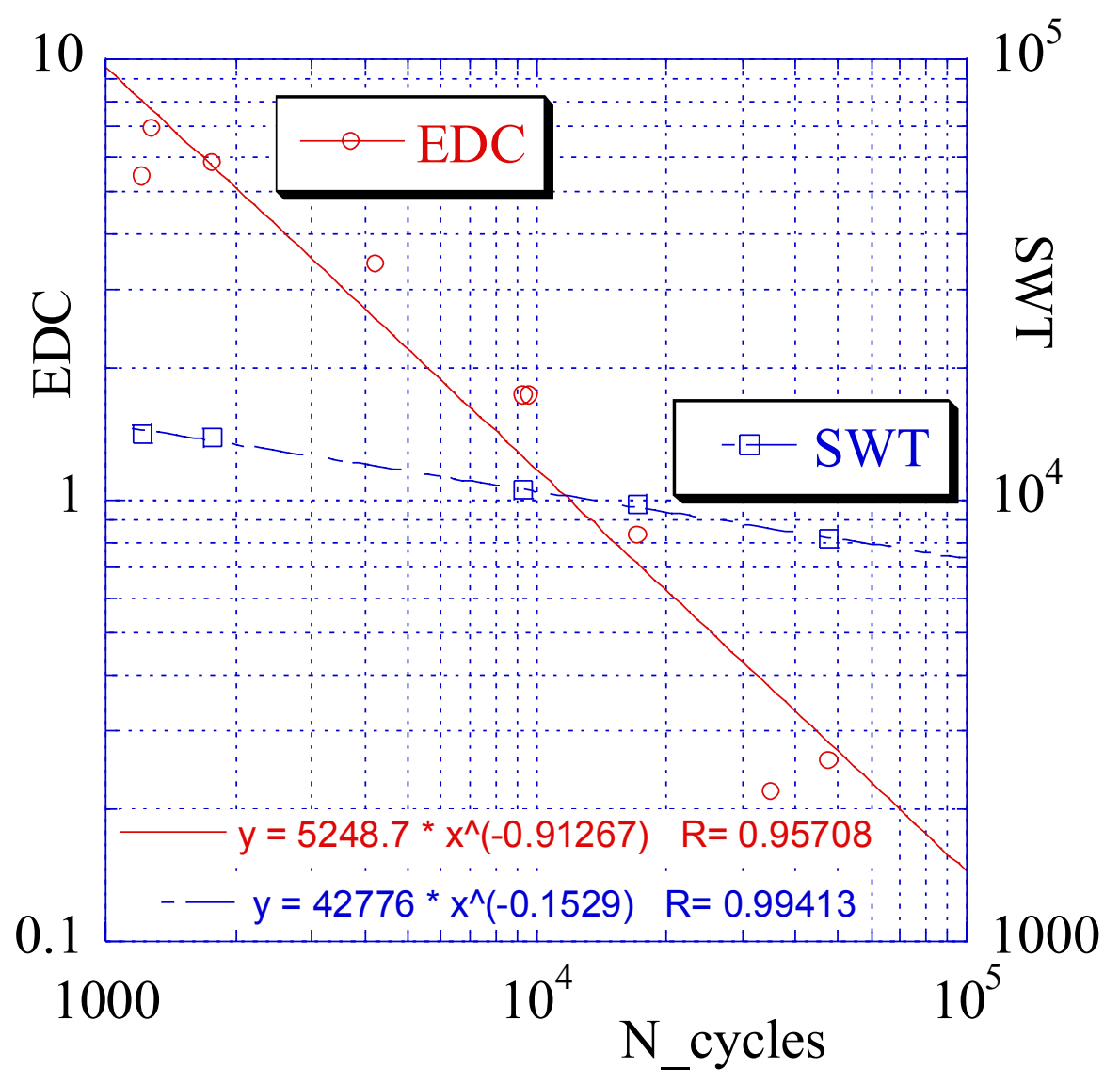

Figure 3. 


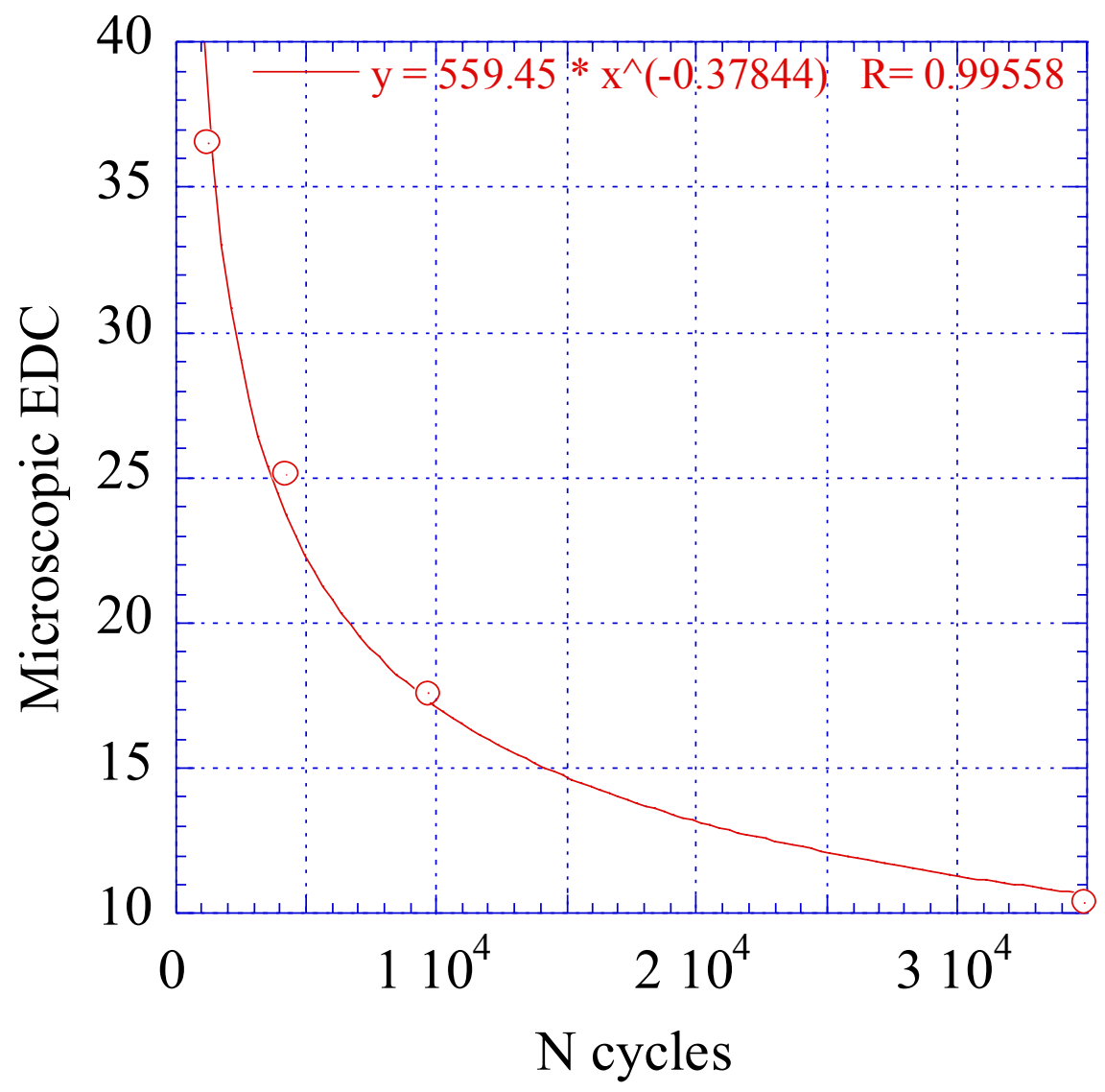

Figure 4. 